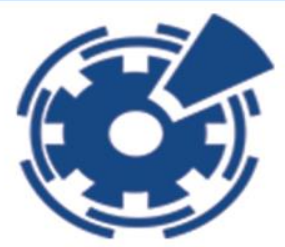

26-28 July, 2019 London, United Kingdom

\title{
Innovative Catalyst to Produce N-Benzylaniline For Drug Applications
}

\author{
Ahmad Alshammari ${ }^{1}$, Rashid Altamimi ${ }^{2}$ and Rajenahally V. Jagadeesh ${ }^{3}$ \\ 1,2 Materials Science Research Institute (MSRI), King Abdulaziz City for Science and Technology (KACST), P.O. \\ Box 6086, Riyadh 1442, KSA \\ ${ }^{3}$ Leibniz-Institut für Katalyse e. V. an der Universität Rostock, Albert Einstein-Str. 29a, 18059 Rostock, Germany
}

\begin{abstract}
Innovations that promote the efficient manufacture of the key ingredients in drugs are recognised in the pharmaceutical industry as conferring considerable benefits to the cost, efficacy and quality of products, thereby raising the satisfaction of the end users. We report the expedient production of N-benzylaniline (NBA) which undoubtedly demonstrates its extreme importance to manufacture several drugs for respiratory diseases (e.g. asthma, chronic, lung cancers, tuberculosis, etc.). Presently, the production of NBA is a complicated and costly process. The synthesis of NBA is dependent upon a number of raw materials and involves many steps, which adversely affects the price of the end product. Therefore, the potential gains that can be attained by developing novel materials that minimise the complexity and expense of NBA are worthy of pursuit. Therefore, the present study involves the development of novel Pd catalyst to obtain NBA in single step process. Various simple and substituted aromatic, heterocyclic and aliphatic amines were reacted with benzyl alcohol to produce their corresponding N-benzylated amines in good to excellent yields (up to 95\% Yield of NBA). Noteworthy, obtained Pd-catalyst preparation has been up-scaled to kilogram scale and catalysts prepared in both small (mg and grams) and large-scale (kilograms) exhibited similar structural features and activity.
\end{abstract}

Keywords: Pd-nanoparticles, $\mathrm{N}$-alkylation, $\mathrm{H}_{2}$ - borrowing technology, amines, alcohols 